\title{
Croatian diatomites and their possible application as a natural insecticide
}

\author{
Ines Galović ${ }^{1}$, Josip Halamić ${ }^{1}$, Anita Grizelj ${ }^{1}$, Vlatka Rozman², Anita Liška ${ }^{2}$, Zlatko Korunić ${ }^{3}$, \\ Pavo Lucić ${ }^{2}$ and Renata Baličević ${ }^{2}$ \\ ${ }^{1}$ Croatian Geological Survey, Department of Geology, Sachsova 2, 10000 Zagreb, Croatia; (ingalovic@hgi-cgs.hr) \\ 2 University of Josip Juraj Strossmayer in Osijek, Faculty of Agriculture in Osijek, Vladimira Preloga 1, 31000 Osijek, Croatia \\ ${ }^{3}$ Diatom Research and Consulting Inc., 14 Tidefal Dr. Toronto, ON, MW 1J2, Canada
}

doi:10.4154/gc.2017.04

Article history:

Manuscript received November 17, 2016 Revised manuscript accepted February 24, 2017 Available online February 28, 2017
Keywords: diatomite, palaeontology, mineralogy, geochemistry, natural insecticide, mid-Miocene, Croatia

\begin{abstract}
In recent decades, there has been an increase in the use of diatomaceous earth (DE) as a natural insecticide because of its low mammalian toxicity, worker safety, low risk of food residues and the occurrence of resistant insect populations associated with the use of chemical insecticides. Therefore there is potential for research into known but previously undescribed Croatian midMiocene marine diatomites from the perspective of their potential as proper DE that could be mixed with plant extracts as a new formulation for grain storage protection. The marine diatomites belong to the Paratethyan near shore environment, deposited in the upwelling zone during a mid-Miocene temperate climate. Palaeontological, mineral and geochemical analyses were done on ten promising marly sediments from 26 outcrops and one borehole from the North Croatian Basin. The most important ingredient of diatomaceous sediments is silica (biogenic opal-A and $\mathrm{SiO}_{2}$ bound in other silicate minerals including quartz, clay minerals, micas, etc.). The amorphous silica content of the tested Croatian diatomites is relatively low $(\leq 50 \%)$ in comparison with the Celatom ${ }^{\circledR}$ MN 51 standard (medium to high efficient DE) (73.6\%), nevertheless they show in some part even slightly better efficacy against insects. It seems that the enhanced content of smectite in diatomaceous sediments also influences increased absorption of DE. Based on palaeontological results, the most efficient diatomites from the Podsusedsko Dolje and Markuševec (Medvednica Mt.) consist of the mid-sized planktonic Coscinodiscus group of species where Thalassionema nitzschioides dominate and is positively correlated with their absorption. The usage of Boströms' standard formula for getting opal-A from geochemical data was abandoned because of negative results and the modified Murdmaas' formula for hemipelagic sediments was applied. Preliminary results on the aforementioned diatomite (as inert dusts) show good efficacy against tested insects Sitophilus oryzae (LINNAEUS), Tribolium castaneum (HERBST) and Rhyzopertha dominica (FABRICIUS).
\end{abstract}

\section{INTRODUCTION}

Diatomite is a porous, lightweight, siliceous sedimentary rock mainly composed of diatom skeletons formed in marine to freshwater environments of mid to high latitudes and contains $>30 \%$ amorphous opaline silica $\left(\mathrm{SiO}_{2} \cdot \mathrm{nH}_{2} \mathrm{O}\right)$. Commercial diatomite contains $>60 \%$ of biogenic silica (BSi). Mostly high-grade diatomite $(>80 \% \mathrm{BSi})$ is used as diatomaceous earth (DE), crushed and oven-dried inert dust with a minor content of calcium carbonate, volcanic glass and terrigenous particles (HARWOOD, 2010 ). Sediments that contains $<30 \%$ of diatoms belongs to the tripolite.

Biogenic silica is non toxic to mammals and has been registered in many countries as a food additive. DE is known as a potentially useful grain protectant because it is safe to use, does not affect grain end-use quality, provides long-term protection and is comparable in cost to other methods of grain protection (KORUNIĆ, 2013). Ideally, active DE should have a high BSi content with a uniform particle size (less than 10 microns), a high oil sorption capacity, a large active surface, and very little clay and other impurities (KORUNIĆ, 1998). Lately, there are numerous reports from around the world on the widespread resistance of several stored-product insects to grain protectants from the groups of synthetic pyrethroids, organophosphates, carbonates, chlorinated hydrocarbons, Bacilus thuringiensis, botanicals and chemicals (SUBRAMANYAM \& HAGSTRUM, 1995; KLJAJIĆ \& PERIĆ, 2005). Since only a physical method of controlling insects is involved in DE, genetic resistance is generally unlikely according to EBELING (1971). However, a potential tolerance or even resistance should be considered when choosing DE for replacing existing grain protectants (KORUNIĆ, 1998; RIGAUX et al., 2001; FIELDS, 2003; VAYIAS et al., 2008).

The efficacy of DE against insects depends on the different properties of the diatom particles, including physical and morphological characteristics of diatoms, rather than on its origin. The physical property of diatomite is in its specific gravity (density) of diatom frustules (skeletons), which is about half that of water. The perforations (pores and striae) and open structure of diatom frustules renders diatomite a considerably lower density $(0.12-$ $\left.0.25 \mathrm{~g} / \mathrm{cm}^{3}\right)$ and high porosity (75-85\%), able to absorb and hold up to 3.5 times its own weight in liquid i.e. in this case wax. Wellknown diatoms with pores (areolas) have the physical ability to absorb wax (lipids) from the insects' epicuticle, causing its dehydration and death (EBELING, 1971; KORUNIĆ, 1998), according to high absorbency which speeds up the incorporation of oil.

The earliest known Croatian diatomites are from the Podsusedsko Dolje outcrops (Medvednica Mt.), and can be described as diatomaceous tripolite (outcrops J1-J4: PANTOCSEK, 1903 


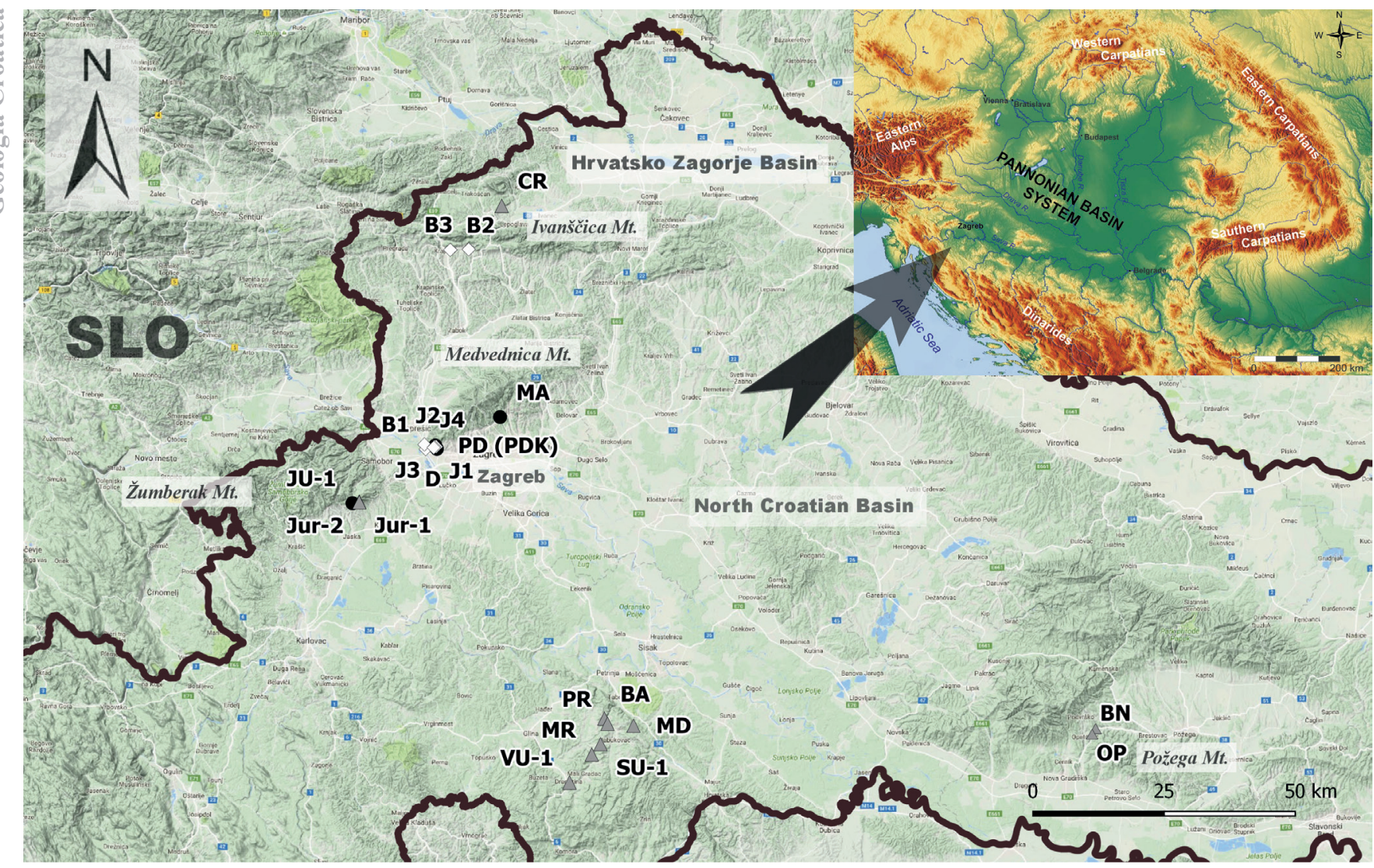

Figure 1. Locations of investigated diatomites in Croatia, Pannonian Basin System based on http://maps-for-free.com: $\diamond-J 1-J 4$ (1903 to 1957) and B1-B3 (1981 to 1984); - Basic Geological Map of Republic of Croatia 1:50.000 from 2000 to 2009; $\boldsymbol{\Delta}$ - this investigations with explanations in the text.

and JURILJ, 1957; outcrop B1: KOCHANSKY-DEVIDÉ \& BAJRAKTAREVIĆ, 1981; outcrops PD-MA: GALOVIĆ \& BAJRAKTAREVIĆ, 2006), and Lopatice and Gornja Šemnica in Hrvatsko Zagorje (outcrops B2-B3: BAJRAKTAREVIĆ, 1984) (Fig. 1). Tripolite is a broad term, used in Croatia for laminated marly sediments by many of the aforementioned authors. The first applied terminology of diatomite was used by GORJANOVIĆKRAMBERGER (1908) and JURILJ (1957). During the investigations for the Croatian Basic Geological Map 1:50.000 from 2000 to 2004, a few more outcrops of diatomaceous tripolite (i.e. diatomite) were discovered in Hrvatsko Zagorje (CR), at Medvednica, Žumberak (Jur-1 and Jur-2) and Požeška gora Mts. Seventyseven more diatom taxa were determined during those investigations on the Podsusedsko Dolje outcrops, where 23 were hitherto not known for the Sarmatian (GALOVIĆ \& BAJRAKTAREVIĆ, 2006).

Our investigations are part of the Croatian Science Foundation (HRZZ) project „Development of new natural insecticide formulations based on inert dusts and botanicals to replace synthetic, conventional insecticides" - DIACROMIXPEST conducted from 2014-2016 (ROZMAN et al., 2015). Throughout this research six more outcrops of diatomite have been discovered in the Banovina region, two in Slavonia and one at Žumberak Mt. (Fig. 1). The palaeontological, mineralogical and geochemical results of Croatian diatomites are presented here with their potential usage as DE in agriculture for the protection of stored products.

\section{GEOLOGICAL SETTING}

The investigated mid-Miocene (Sarmatian) sediments of the North Croatian Basin (NCB) and Hrvatsko Zagorje Basin (HZB), belong to the south-western marginal part of the Pannonian Basin
System (PBS) (Central Paratethys) (PAVELIĆ, 2001; PAVELIĆ et. al., 2003) (Fig. 1). This intracontinental region began to develop in the early Miocene as a result of the African and European plate collision (HORVÁTH \& ROYDEN, 1981). PBS is surrounded by the Alps, Dinarides and Carpathian Mountains. The evolution of the Central Paratethys was controlled by regional tectonic events, sea-level changes and connections with the Mediterranean, Boreal and Indo-Pacific Oceans (STEININGER et al., 1988; RÖGL, 1996). These connections have been repeatedly disrupted and re-established until the end of the Badenian when the sea level dropped, causing the beginning of the isolation and disintegration of the Paratethys. At the beginning of the Sarmatian, the connection with the other marine areas further weakened, and was followed by isolation; a stronger terrestrial influence favoured further decrease in salinity, resulting in changes to flora and fauna and their endemism. This was all more strongly influenced by climate changes. From the warmer period in the Badenian, the climate during the Sarmatian becomes more moderate with seasonal oscillations (GALOVIĆ, 2001). In the Sarmatian, the connections with the Mediterranean and Indo-Pacific realms also existed, as shown by the last Miocene transgression (RÖGL, 1998; KOVÁČ et al., 2001), and corroborated by investigations of siliceous microplankton (JURILJ, 1957; HAJÓS, 1986; GALOVIĆ \& BAJRAKTAREVIĆ, 2006). The total thickness of the Sarmatian deposits is relatively small (50 to $150 \mathrm{~m}$ ), they predominantly consist of marly sediments of the tripolite type (BAJRAKTAREVIĆ et al., 1986) with rare limestones, clastic and volcanic fragments. Such a small thickness is probably a consequence of the basin deepening (due to lithosphere cooling and compression at the end of the Sarmatian) and less sediment input, which is characteristic of the early post-rifting phase (PAVELIĆ et al., 2003). 
STAGE

OUTCROPS
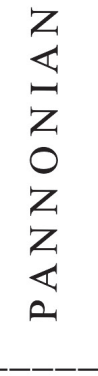

\section{Podsusedsko Dolje}

«
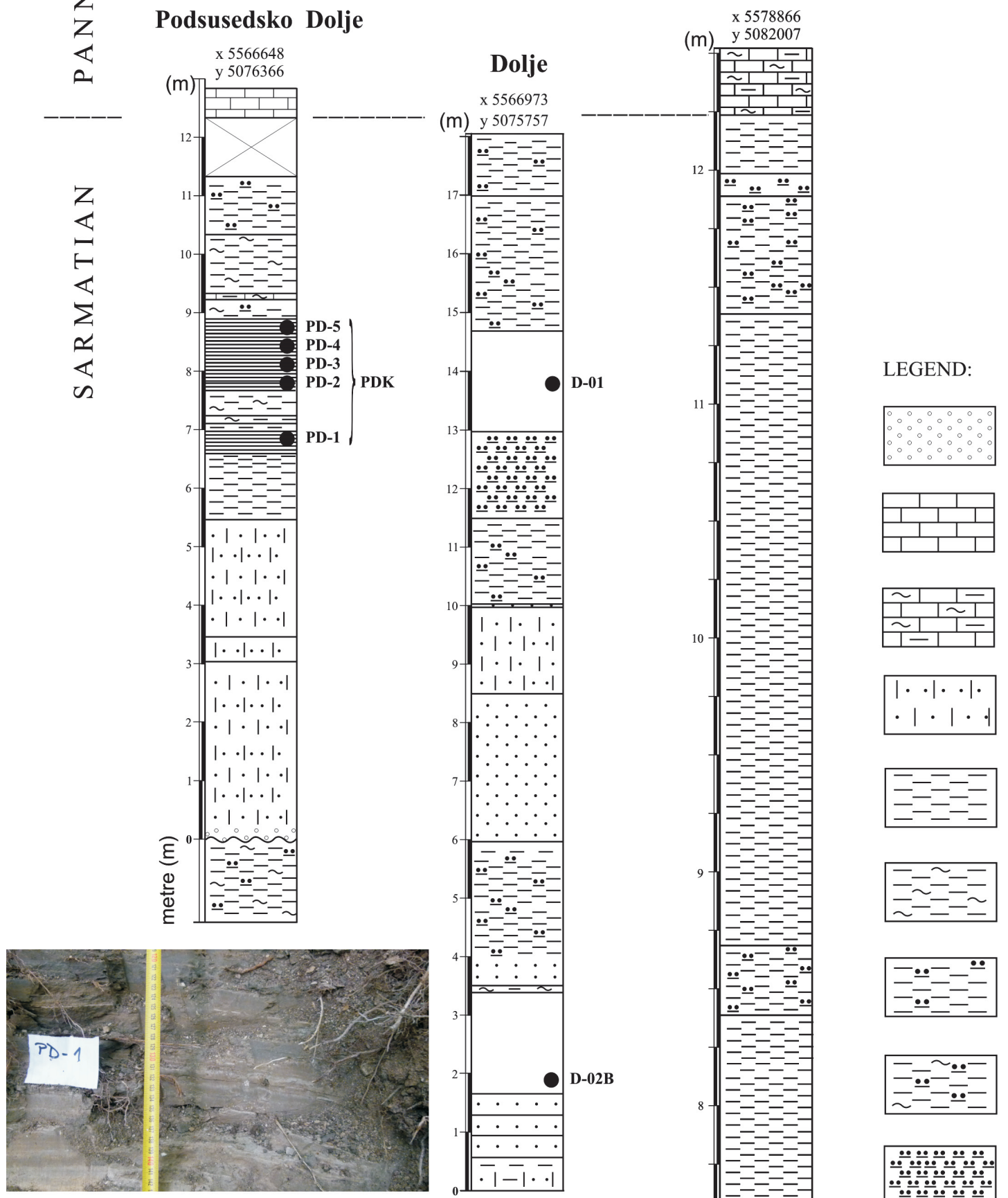

CONGLOMERATE

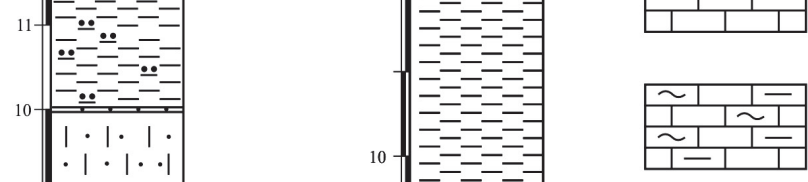

LIMESTONE

CLAYEY

LIMESTONE

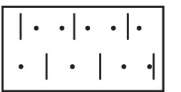

SPICULITE

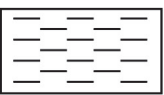

MARL

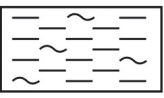

CLAYEY MARL

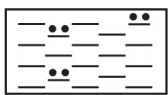

SILTY MARL

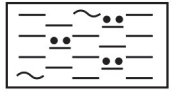

SILTY-CLEYEY

MARL

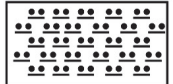

SILTSTONE
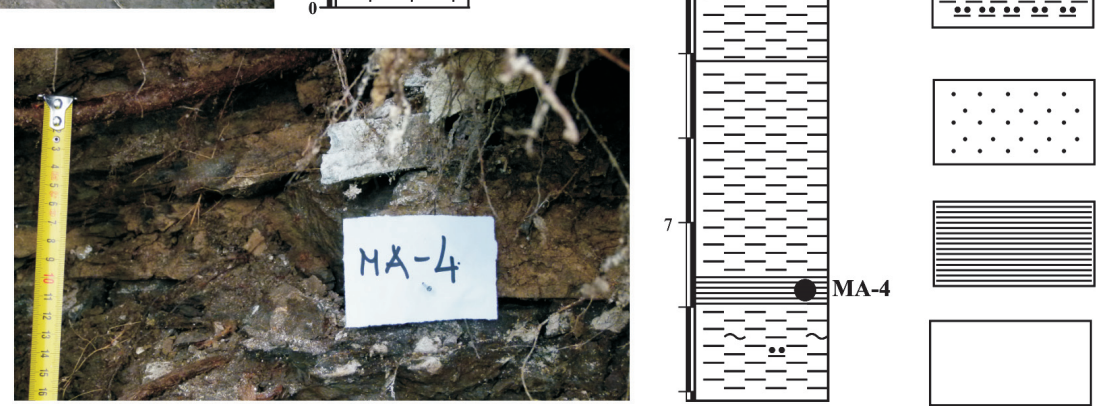

SANDSTONE

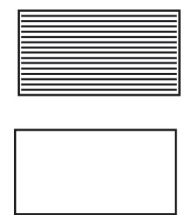

TRIPOLITE

DIATOMITE

Figure 2. The parts of the geological outcrops from Medvednica Mt. where the most effective Croatian diatomite and tripolite were found. 
Since the beginning of the Miocene (23 Ma; OGG et al., 2016), the most commercially exploited diatomaceous earth deposits are lacustrine in origin, although the largest producing deposit that outcrops near Lompoc in California is a marine deposit of Miocene age (DOLLEY \& MOYLE, 2003). Nevertheless, other diatomites from Paratethys are also marine deposits of Miocene age from Slovenia, Austria, Czech, Slovakia, Hungary and Serbia (ŘEHÁKOVÁ, 1977; HAJÓS, 1986; HORVAT, 2004; ROETZEL et al., 2006; SCHÜTZ et al., 2007; ROJHT et al., 2010). Well known Croatian diatomites from Medvednica Mt. belong to near shore mid-Sarmatian marine sediments characteristic of the upwelling zone (GALOVIĆ \& BAJRAKTAREVIĆ, 2006).

Individual parts of the NCB were uplifted and thus became source regions for clastic Sarmatian deposits, accumulating in environments of reduced salinity (VRSALJKO, 1999). Through advanced ingression and deepening of the depositional environment, thin-bedded to laminated marls and sandstones accumulated (VRSALJKO et al., 2005; VRSALJKO, 1997).

In the NCB Sarmatian shallow-water gravels, biocalcarenites, and limestones were laid down, whereas later laminated marly deposits predominated, due to the deepening of the basin. For the region of the Žumberak and Samobor Mts., Sarmatian sediments consisting of calcite-rich marls and limestones with sandstone intercalations, crop out in a narrow zone along the Badenian deposits, (VRSALJKO, 2003).

\section{MATERIALS AND METHODS}

\subsection{Field research with laboratory tests}

The representative sediments for diatom analyses belong to marl, tripolite, laminated marls, diatomite, varve, silt, clay and their varieties (Fig. 2). The thicknesses of the sampled diatomites are small, approx. $48 \mathrm{~cm}$. They are mostly laminated as a result of seasonality. Among 26 well known Sarmatian outcrops of diatomites in Croatia, twelve were selected for further investigation: Baničevac (BN) and Opatovac (OP) in Slavonia, Jurjevčani (JU) at Žumberak Mt., Crkovec (CR) in Hrvatsko Zagorje, Markuševec (MA) and Podsusedsko Dolje (PD) at Medvednica Mt. and Bačuga (BA), Martinovići (MR), Prnjavor Čuntički (PR), Šušnjar (SU), Mali Deanovići (MD) and Vukičevići (VU) in Banovina. Samples from the Dolje borehole at Podsusedsko Dolje were also examined (D) (ROZMAN et al., 2016) (Fig.1). In total, 57 samples (each of ca. $10 \mathrm{~kg}$ ) were taken for preliminary testing on their insecticidal efficacy by comparing them with standard medium to high effective Celatom ${ }^{\circledR}$ MN 51. After testing, ten samples showed promising insecticidal efficacy and were selected for further analyses: MA-4, PD-1, PDK, D-01 and D-02B, JU-1, OP-4 and OP-4A (less laminated marls from the same horizont), MR10 (siltic marl) and MR-10B (mm laminated marl) and examined as natural pesticide (LIŠKA et al., 2015). Sample PDK is mixture of all five PD samples, but milled to a diameter $<45 \mu \mathrm{m}$ for testing efficacy with smaller particles in the second year of the research project. Based on their preliminary physico-chemical properties (palaeontological, mineral and geochemical analyses) only samples from Medvednica Mt. (Fig. 2) were affirmed as diatomite.

\subsection{Palaeontology}

The standard preparation method used by the Croatian Geological Survey is applied. Approximately $1 \mathrm{~cm}^{3}$ of the sediment is placed in a beaker and treated with $30 \%$ concentrated hydrogen peroxide $\left(\mathrm{H}_{2} \mathrm{O}_{2}\right)$ to oxidize organic matter for light microscope (LM) analyses. Siliceous microfossil slides for SEM preparations also include treatment with $20 \%$ of concentrated $\mathrm{HCl}$ acid to remove carbonate and $10 \%$ of sodium pyrophosphate to remove clay components. The sediments are then rinsed with distilled water. Some of the samples were treated in an ultrasonic bath for approximately 15 seconds to improve disaggregation. Standard smear slides and SEM stub preparation techniques were used in slide preparation for both LM and SEM analysis. The specimens were coated with gold and studied with a JEOL JSM-35CF SEM. Smear slides were examined using a BH2 Olympus LM.

Composition and diversity of diatom species together with their morphometric analyses are essential for the determination of DE quality. Quantitative methods were undertaken for 300 valves of diatom genera along transects under $500 \mathrm{x}$ magnification (VILIČIĆ, 2003; HORVAT, 2004). Only whole diatom valves or their representative parts are counted according to SCHRADER \& GERSONDE (1978) and HORVAT (2004). Diatom measurements were made using free ImageJ software (https://imagej.nih. gov/ij/).

Determined diatoms are traditionally divided into two orders: Centrales (radial symmetry) and Pennales (bilateral symmetry).

\subsection{Mineralogy}

X-ray powder diffraction (XRPD) was recorded on bulk samples, insoluble rock residue and the $<2 \mu \mathrm{m}$ fraction of samples. Some samples contained large amounts of carbonate minerals that had to be dissolved to eliminate overlapping diffraction peaks with other minerals. Carbonate fractions were dissolved by acetic acid with an ammonium acetate $\left(1 \mathrm{~mol} \mathrm{dm}^{-3}\right)$ buffer of $\mathrm{pH} 5$ (JACK$\mathrm{SON}, 1956)$. Clay minerals were determined on the $<2 \mu \mathrm{m}$ fraction of samples on oriented mounts of air dried material, and after glycol treatment, heating to $400{ }^{\circ} \mathrm{C}$ and $550{ }^{\circ} \mathrm{C}$ (STARKEY et al., 1984). For quantitative analyses samples with $10 \%$ internal standard (zincite) were carefully weighed and ground with about $4 \mathrm{ml}$ of methanol in a McCrone mill.

For XRPD analysis a Philips vertical X-ray goniometer (type $\mathrm{X}^{\prime}$ Pert) equipped with a $\mathrm{Cu}$ tube at the Croatian Geological Survey (Zagreb, Croatia) was used. Experimental conditions were: $45 \mathrm{kV}, 40 \mathrm{~mA}$, PW 3018/00 PIXcel detector, primary beam divergence $1 / 4^{\circ}$, and continuous scan $\left(0.02^{\circ} 2 \Theta / s\right)$.

Quantitative analyses of the insoluble residue of samples were made using the RockJock ${ }^{\circledR}$ computer program (EBERL, 2003).

\subsection{Geochemistry}

For better understanding of their mineralogical composition all selected samples were also analysed by XRF methods for the major oxides and some trace elements as $\mathrm{Ba}, \mathrm{Cu}, \mathrm{Ni}, \mathrm{Pb}, \mathrm{Sr}, \mathrm{V}_{2} \mathrm{O}_{5}$, $\mathrm{Zn}$, and $\mathrm{Zr}$ in laboratories of the Bureau Veritas Commodities Ltd (Vancouver, Canada). A $5 \mathrm{~g}$ of sample was heated to determine the loss on ignition (LOI). The treated sample was then fused in a platinum-gold crucible with a lithium tetraborate. The molten material is cast in a platinum mold. Fused discs were analysed on PANalytical Axios ${ }^{\mathrm{mAX}}$.

The inductively coupled plasma atomic emission spectrometry/inductively coupled plasma emission mass spectrometry (ICP-AES / ICP-MS) methods for measuring trace elements were performed on $15 \mathrm{~g}$ samples. The $0.25 \mathrm{~g}$ split were heated in $\mathrm{HNO}_{3}-\mathrm{HClO}_{4}-\mathrm{HF}$ to fuming and taken to dryness. The residue was dissolved in $\mathrm{HCl}$. This preparation method is only partial for some $\mathrm{Cr}$ and $\mathrm{Ba}$ minerals and oxides of $\mathrm{Al}, \mathrm{Fe}, \mathrm{Hf}, \mathrm{Mn}, \mathrm{Sn}, \mathrm{Ta}$ 
Table 1. Diatom distribution with their morphometrics. Legend: + scattered (< $5 \%)$, rare (5-9\%), low abundance (10-15\%), abundant (16-35\%), high abundance (36-50\%), dominant species (>50\%) - species in fragments.

\begin{tabular}{|c|c|c|c|c|c|c|c|}
\hline Diatom/sample & PD-1 & D-01 & $\mathrm{D}-02 \mathrm{~B}$ & MA-4 & JU-1 & PDK & size $(\mu \mathrm{m})$ \\
\hline \multicolumn{8}{|l|}{ Centrales } \\
\hline Actinocyclus octanarius EHRENBERG & & + & + & & & + & $8-35$ \\
\hline Actinocyclus octanarius var. tenellus (BREB.) HENDEY & & + & & & + & + & $25-60$ \\
\hline Actinoptychus senarius (EHRENBERG) EHRENBERG & & + & + & & & + & $20-62$ \\
\hline Anaulus minutus GRUNOW & + & & & & & + & $10-16$ \\
\hline Anaulus simplex HAJÓS & + & & + & & + & + & $8-12$ \\
\hline Asteromphalus brunii PANTOCSEK & & + & & & & & $32-44$ \\
\hline Coscinodiscus group (Coscinodiscus, Hyalodiscus, Melosira, Paralia, Stephanopyxis, Thalassiosira) & + & + & 22 & 25 & 16 & 27 & \\
\hline Coscinodiscus apiculatus EHRENBERG & & & + & & & & - \\
\hline Coscinodiscus curvatulus GRUNOW & + & + & 8 & + & & + & 19- 37 \\
\hline Coscinodiscus doljensis PANTOCSEK & & + & + & 17 & + & + & $28-43$ \\
\hline Coscinodiscus miocaenicus KRASSKE & & + & & + & & & $18-21$ \\
\hline Coscinodiscus obscurus SCHMIDT & & & + & & & & 38 \\
\hline Coscinodiscus oculus iridis EHRENBERG & + & & + & + & + & + & $\geq 40-75$ \\
\hline Coscinodiscus perforatus var. cellulosus GRUNOW & & & + & & & + & - \\
\hline Coscinodiscus radiatus EHRENBERG & & + & + & & & & $68-72$ \\
\hline Coscinodiscus rothii (EHRENBERG) GRUNOW & & + & + & + & & & $39-80$ \\
\hline Coscinodiscus rugulosus HAJÓS & & + & + & & & & $14-30$ \\
\hline Coscinodiscus sarmaticus PANTOCSEK & & & + & + & + & + & $12-31$ \\
\hline Hemiaulus cf. polymorphus & & & & & & + & 46 \\
\hline Hyalodiscus laevis EHRENBERG & & & & & & + & $20-50$ \\
\hline Hyalodiscus scoticus (KÜTZING) GRUNOW & & & & & + & + & $10-40$ \\
\hline Melosira dickiei var. fossilis PANTOCSEK & & & + & & & & $12-20$ \\
\hline Melosirasp. & & & & & + & & - \\
\hline Paralia sulcata (EHRENBERG) CLEVE & + & + & + & + & + & 7 & $12-40$ \\
\hline Perissonoë trigona (GRUNOW) ANDREWS \& STOELZEL & & & & & & + & - \\
\hline Pseudopodosira westii (SMITH) SHESHUKOVA-PORETZSKAYA \& GLEZER & & & + & + & & + & $12-29$ \\
\hline Rhizosolenia oligocaenica SCHRADER & & + & + & + & + & + & - \\
\hline Stellarima stellaris (ROPER) HASLE \& SIMS & + & + & + & + & + & & 36 \\
\hline Stephanopyxis turris (GREVILLE) RALFS & + & & + & & & & 63 \\
\hline Thalassiosira leptopus (GRUNOW) HASLE \& FRYXELL & + & + & + & + & + & + & $34-65$ \\
\hline $\begin{array}{l}\text { resting spores and siliceous cysts (Bacteriastrum spp., Chaetoceros spp, Liradiscus spp., } \\
\text { Xantiopyxis spp., Periptera spp.) }\end{array}$ & 6 & 59 & + & + & 6 & 6 & - \\
\hline Triceratium balearicum CLEVE \& GRUNOW & & & & & & + & - \\
\hline T. laetum fa. quadrata HAJÓS & & & & & & + & 28 \\
\hline \multicolumn{8}{|l|}{ Pennales } \\
\hline Achnanthes baldjiki (BRIGHTW.) GRUNOW & & & & & & + & 32 \\
\hline Achnanthes brevipes AGARDH & & + & & & & & 31 \\
\hline Achnanthes rara JURILJ & & + & & & & + & $29-34$ \\
\hline Achnanthes saeptata var. sussedana JURILJ & & & & & + & & 50 \\
\hline Achnanthes spp. & & & & & + & & - \\
\hline Amphora binodis var. biggiba GRUNOW & + & & & & & + & 28 \\
\hline Amphora costata SMITH & + & & & & & + & 63 \\
\hline Ardisonia fulgens (GREVILLE) GRUNOW & & & & & & + & - \\
\hline Biddulphia biddulphiana (BOYER) SMITH & & & + & & + & & $54-117$ \\
\hline Biddulphia sp. & & & & & & + & - \\
\hline Caloneis boryana PANTOCSEK & & & & & & + & 42 \\
\hline Climacosphenia moniligera EHRENBERG & & & & & & + & $16-18$ \\
\hline Cocconeis andesitica PANTOCSEK & & & & & & + & 30 \\
\hline C. canaliculata JURILJ & & & & & & + & 15 \\
\hline Cocconeis conciata PANTOCSEK & + & & & & & + & 26 \\
\hline C. disculus (SCHUMANN) CLEVE & & & & & & + & 14 \\
\hline C. distans GREGORY & & & & & & + & 40 \\
\hline C. evolvens JURILJ & & & & & & + & 23 \\
\hline Cocconeis fluminensis (GRUNOW) PERAGALLO & + & & & & & + & 28 \\
\hline C. quarnerensis var. lanceolata JURILJ & & & & & & + & 20 \\
\hline Cocconeis cf. sarmatica PANTOCSEK & + & & & & & + & $11-28$ \\
\hline Cocconeis scutellum EHRENBERG & + & + & + & + & + & 5 & $9-45$ \\
\hline Cymatosira lorenziana var. maior JURILJ & + & & & & & + & $34-54$ \\
\hline Delphineis angustata (PANTOCSEK) ANDREWS & & + & & & & & $20-35$ \\
\hline Delphineis surirella (EHRENBERG) ANDREWS & & + & + & & & & 20 \\
\hline Dimeregramma angustatum HAJÓS & & & & & 5 & & 40 \\
\hline
\end{tabular}


Table 1. continued

\begin{tabular}{|c|c|c|c|c|c|c|c|}
\hline Diatom/sample & PD-1 & D-01 & D-02B & MA-4 & JU-1 & PDK & size $(\mu \mathrm{m})$ \\
\hline \multicolumn{8}{|l|}{ Centrales } \\
\hline Dimerogramma boryanum PANTOCSEK & & & & & & + & - \\
\hline Dimeregramma distans GREGORY & & & & & + & & 39 \\
\hline Di. minor (GREGORY) RALFS & & & & & & + & $12-17$ \\
\hline Di. minus var. neglectum JURILJ & & & & & & + & - \\
\hline Diploneis bombus EHRENBERG & & & & & + & & - \\
\hline Diploneis coffaeiformis (SCHMIDT) CLEVE & & & & & & + & - \\
\hline Diploneis crabro (EHRENBERG) EHRENBERG & & & + & + & & + & $22-45$ \\
\hline Diploneis fusca (GREGORY) CLEVE & + & & & & & + & 51 \\
\hline Diploneis ovalis (BRÉBISSON) CLEVE & + & + & + & + & & + & 34 \\
\hline Diploneis splendida var. porosa JURILJ & & & & + & & & - \\
\hline Diploneis subovalis CLEVE & & & + & & & + & $43-57$ \\
\hline Diploneis smithii (BRÉBISSON) CLEVE & + & & & & + & + & $30-50$ \\
\hline Diploneis sp. & & + & & & & & - \\
\hline Encyonemasp. & & & & & & + & - \\
\hline Epithemia adnata (KÜTZING) BRÉBISSON & & & & & + & & 46 \\
\hline Epithemia zebra var. saxonica (KÜTZING) GRUNOW & & & & & & + & - \\
\hline Eunotia cf. tenella (GRUNOW) HUSTEDT & & & & & & + & - \\
\hline Fragilaria brevistriata GRUNOW & + & & & + & & + & $9-15$ \\
\hline Fragilaria construens (EHRENBERG) GRUNOW & & & + & & & + & 8 \\
\hline Grammatophora angulosa EHRENBERG & & & & + & & + & $28-32$ \\
\hline Grammatophora insignis GRUNOW & + & & & & + & + & - \\
\hline Grammatophora macilenta SMITH & & & & & + & & $25-80$ \\
\hline Grammatophora miocaenica HAJÓs & & + & & & & & 28 \\
\hline Grammatophora robusta EHRENBERG & & & & + & & & $40-60$ \\
\hline Grammatophora oceanica EHRENBERG & & + & + & & & + & $37-93$ \\
\hline G. oceanica var. macilenta (SM.) GRUNOW & & & & & & + & do 172 \\
\hline G. oceanica var. oceanica EHRENBERG & & & & & & + & - \\
\hline Grammatophora stricta EHRENBERG & + & + & & & + & + & $22-40$ \\
\hline G. stricta var. biharensis EHRENBERG & & & & & & + & - \\
\hline Hantzschia virgata (ROPER) GRUNOW & & & & & + & & $50-66$ \\
\hline Lyrella hennedyi (W.SMITH)STICKLE \& MANN & + & + & & & & + & $25-45$ \\
\hline Mastogloia binotata (GRUNOW) CLEVE & & & & & + & & 40 \\
\hline Mastogloia lacustris (GRUNOW) GRUNOW & & & + & & + & & 38 \\
\hline Mastogloia sarmatica JURILJ & + & & + & & & + & 69 \\
\hline M. splendida (GREGORY) CLEVE & & & & & & + & - \\
\hline Mastogloia sp. & + & + & + & & 9 & & - \\
\hline Navicula latissima var. quadrata JURILJ & & & & + & & + & 64 \\
\hline Navicula spp. & & + & & & + & & - \\
\hline Nitzschia doljensis PANTOCSEK & & + & & & & & 35 \\
\hline Nitzschia frustulum (KÜTZING) GRUNOW & & & + & + & & & 18 \\
\hline Nitzschia spp. & + & & & & + & & - \\
\hline Opephora gemmata fa. minor JURILJ & & & & & + & & 40 \\
\hline Opephora marina (GREGORY) PETIT & & & & & & + & 17 \\
\hline Plagiogramma biharense PANTOCSEK & & & + & & & & 15 \\
\hline Plagiogramma bipunctatum HAJÓS & + & & & & & & - \\
\hline Plagiogramma staurophorum (GREGORY) HEIBERG & + & & + & & + & + & $20-30$ \\
\hline Planothidium quarnerensis (GRUNOW) WITKOWSKI,LANGE-BERTALOT \& METZELIN & & & & & + & + & 20 \\
\hline Rhabdonema hamuliferum KITTON & & & & & 12 & & - \\
\hline Rhaphoneis amphiceros EHRENBERG & & & & & + & + & $17-32$ \\
\hline Rh. amphiceros var. rhombica GRUNOW & & & & & & + & - \\
\hline Rh. cocconeiformis (SCHMIDT) HANNA \& GRANT & & & & & & + & - \\
\hline Rh. nitida (GREGORY) GRUNOW & & & & & & + & - \\
\hline Rhaphoneis rhombica (GRUNOW) ANDREWS & + & & & & & + & 34 \\
\hline Rhopalodia gibberula (EHRENBERG) MÜLLER & + & & & & + & + & 34 \\
\hline Staurosirella leptostauron (EHRENBERG) WILLIAMS \& ROUND & & & & & + & & $10-26$ \\
\hline Staurosirella pinnata (EHRENBERG) WILLIAMS \& ROUND & & + & & & & & 22 \\
\hline Surirella subfastuosa PANTOCSEK & & & & & + & & - \\
\hline Synedra crystallina var. fossilis PANTOCSEK & & & & & & + & - \\
\hline Synedra fulgens (GREVILLE) W.SMITH & + & & + & & + & & - \\
\hline Synedra tabulata var. obtusa PANTOCSEK & & & & & + & & - \\
\hline Thalassionema nitzschioides (GRUNOW) GRUNOW & 69 & 32 & 74 & 60 & 30 & 8 & $33-139$ \\
\hline Thalassiotrix longissima CLEVE \& GRUNOW & 8 & & + & + & + & + & 34 \\
\hline
\end{tabular}


and $\mathrm{Zr}$. Additionally, volatization during fuming may result in some loss of As, $\mathrm{S}$ and $\mathrm{Sb}$. The analyses were done by SPECTRO CIRIOS VISION ICP-AES and Perkin Elmer Elan 6000/9000 ICP-MS. Inorganic carbon was determined by directly measuring the $\mathrm{CO}_{2}$ gas evolved into the LECO CS230 analyzer when a prepared sample split is leached with perchloric acid.

From the last century, the Boströms' standard formula (BOSTRÖM at al., 1972) for estimating biogenic silica from geochemical data was used:

$$
\text { Opal- } \mathrm{A}=\mathrm{SiO}_{2}(\text { total })-3 \cdot \mathrm{Al}_{2} \mathrm{O}_{3}
$$

We tested and abandoned this formula because of a negative result for sample MR-10B, (Tab. 5). Therefore Murdmaas' modified formula (MURDMAA et al., 1980) for extracting opal-A from hemipelagic sediments was used:

$$
\text { Opal-A }=\mathrm{SiO}_{2}(\text { total })-2.7 \cdot \mathrm{Al}_{2} \mathrm{O}_{3}
$$

\subsection{Insecticidal efficacy}

In order to test insecticidal efficacy of inert dusts against rice weevil, S. oryzae, 100 grams of clean soft wheat of different varieties (approx. 13\% m.c.) was mixed with a determined quantity of each dust in glass jars of $200 \mathrm{~mL}$. Fifty unsexed adults of $S$. oryzae, 7-21 days old were added into each jar. Inert dusts were tested in 3 or 4 different doses, depending on inert dust, and all treatments were conducted in 4 repetitions. Bioassay was kept under controlled conditions at $28 \pm 2{ }^{\circ} \mathrm{C}, 65 \pm 5 \% \mathrm{RH}$ and in dark.

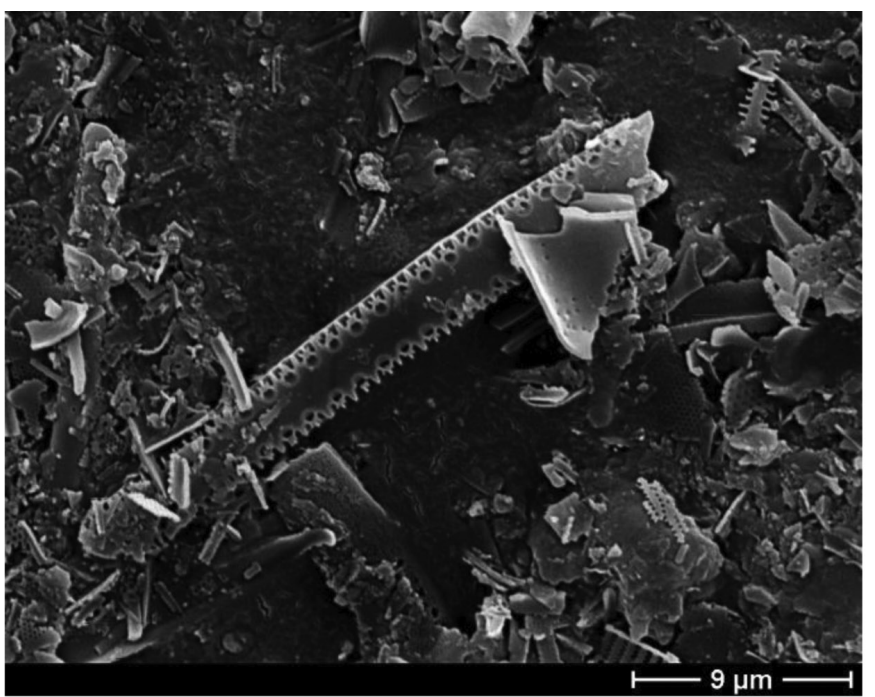

Insecticidal efficacy was estimated as lethal doses required to kill $50 \%$ and $90 \%$ of the tested population $\left(\mathrm{LD}_{50}\right.$ and $\left.\mathrm{LD}_{90}\right)$. The $\mathrm{LD}_{50}$ and $\mathrm{LD}_{90}$ values were calculated by Probit analysis using IBM SPSS Statistics (IBM Corp. Released, 2013).

\section{RESULTS}

\subsection{Palaeontology}

In total, in samples from Medvednica and Žumberak Mts, 111 diatom species were determined with fourteen variants and two forms. Among them, four species had not been previously discovered in Podsusedsko Dolje: Chaetoceros didymus EHRENBERG, Dimerogramma minus (GREGORY) RALFS var. neglectum JURILJ, Rhaphoneis cocconeiformis (SCHMIDT) HANNA $\&$ GRANT and Rh. nitida (GREGORY) GRUNOW. Approximately $90 \%$ of the diatom species are less than $50 \mu \mathrm{m}$ in size, while larger specimens are mostly broken (GALOVIĆ et al., 2015).

Diatom distribution with their sizes is given in Table 1. to emphasise the physical character of diatomite as a potential natural pesticide. The most abundant species in the samples belong to the Coscinodiscus group, Thalassionema nitzschoides and Chaetoceros group with resting spores and siliceous cysts. Based on palaeontological results, the most prospective diatomite (tripolite) for commercial use would be from Medvednica Mt. (MA-4, PDK, D-02B), Fig. 3. The dominant species in sample MA-4 is

Figure 3. SEM images of partially crushed diatom Thalassionema nitzschioides on the left an Coscinodiscus curvatulus on the right from the sample D-02B.

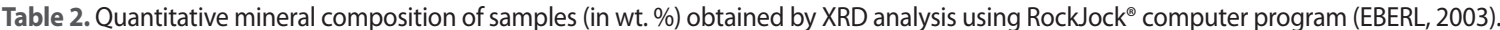

\begin{tabular}{|c|c|c|c|c|c|c|c|c|c|}
\hline MINERALS & $M A-4$ & PDK & D-01 & $D-02 B$ & JU-1 & $\mathrm{OP}-4$ & OP-4A & MR-10 & MR-10B \\
\hline Quartz & 5 & 3 & 5 & 4 & 2 & 2 & 1 & 2 & 2 \\
\hline Opal-A & 28 & 12 & 41 & 50 & 11 & & & 3 & \\
\hline Opal-C & & + & + & & & 1 & 1 & & \\
\hline Calcite & 19 & 30 & 10 & 11 & 28 & 7 & 6 & 69 & 64 \\
\hline Aragonite & & 34 & & & 40 & 73 & 81 & & \\
\hline Feldspar & 1 & 2 & & & 1 & 1 & 1 & 1 & \\
\hline Clay minerals & 47 & 19 & 44 & 33 & 18 & 16 & 10 & 25 & 34 \\
\hline Smectite & $* *$ & * & $* *$ & $* *$ & * & * & * & * & * \\
\hline Illit/muscovite & * & * & * & * & * & * & * & * & * \\
\hline Chlorite & $*$ & & & & & + & & & \\
\hline Kaolinite & & * & & & & + & + & + & + \\
\hline
\end{tabular}
** - abundant $(20-40 \%),{ }^{*}$ - subordinate $(1-20 \%),+-$ traces $(<1 \%)$ 
Table 3. Major and trace elements content measured by XRF method (in \%) in Croatian diatomites and major elements in Celatom ${ }^{\circledR}$ Diatomaceous Earth Functional Additive Celatom ${ }^{\circledR}$ MN 51 standard.

\begin{tabular}{|c|c|c|c|c|c|c|c|c|c|c|c|c|c|c|}
\hline $\begin{array}{l}\text { Sample/ } \\
\text { Element }\end{array}$ & $\mathrm{SiO}_{2}$ & $\mathrm{Al}_{2} \mathrm{O}_{3}$ & $\mathrm{Fe}_{2} \mathrm{O}_{3}$ & $\mathrm{CaO}$ & $\mathrm{MgO}$ & $\mathrm{Na}_{2} \mathrm{O}$ & $\mathrm{K}_{2} \mathrm{O}$ & $\mathrm{MnO}$ & $\mathrm{TiO}_{2}$ & $\mathrm{P}_{2} \mathrm{O}_{5}$ & LOI & SUM $^{* * *}$ of trace elements & SUM & TOT/C \\
\hline $\mathrm{MA}-4$ & $47.71^{*}$ & 8.81 & 3.85 & 9.63 & 0.92 & 0.37 & 1.43 & 0.07 & 0.41 & 0.10 & 23.44 & 2.218 & 98.96 & 9.65 \\
\hline PDK & 21.26 & 4.51 & 1.98 & 36.29 & 0.67 & 0.47 & 0.59 & 0.03 & 0.25 & 0.11 & 33.33 & 0.002 & 99.51 & - \\
\hline D-01 & 55.58 & 6.83 & 2.85 & 5.59 & 1.30 & 0.25 & 1.05 & 0.04 & 0.31 & 0.21 & 22.52 & 2.442 & 98.97 & 8.56 \\
\hline D-02B & 61.91 & 5.31 & 2.20 & 6.13 & 1.13 & 0.19 & 0.82 & 0.02 & 0.27 & 0.06 & 18.60 & 2.326 & 98.97 & 5.83 \\
\hline JU-1 & 18.65 & 3.15 & 1.34 & 38.10 & 0.49 & 0.21 & 0.44 & 0.04 & 0.16 & 0.10 & 35.22 & 1.048 & 98.95 & 10.07 \\
\hline OP-4 & 7.37 & 2.32 & 0.97 & 44.72 & 0.34 & 0.48 & 0.19 & 0.005 & 0.12 & 0.007 & 41.31 & 1.345 & 99.24 & 10.76 \\
\hline OP-4A & 3.96 & 1.31 & 0.40 & 49.26 & 0.24 & 0.39 & 0.09 & 0.02 & 0.07 & 0.11 & 42.78 & 1.105 & 99.74 & 11.61 \\
\hline MR-10 & 14.21 & 4.73 & 1.57 & 37.15 & 1.89 & 0.24 & 0.83 & 0.07 & 0.24 & 0.08 & 36.53 & 1.541 & 99.08 & 10.42 \\
\hline MR-10B & 16.22 & 5.44 & 2.05 & 36.90 & 1.42 & 0.16 & 0.92 & 0.07 & 0.23 & 0.07 & 35.04 & 0.728 & 99.25 & 9.93 \\
\hline Celatom $^{\oplus} \mathrm{MN}^{2} 1^{* *}$ & 73.60 & 7.80 & 1.80 & 5.60 & 0.30 & \multicolumn{3}{|c|}{ SUM other oxides } & 2.30 & - & 5.50 & - & 96.90 & - \\
\hline
\end{tabular}

* $\ln \%$

* Standard DE

*** Measured trace elements: $\mathrm{Ba}, \mathrm{Cu}_{1} \mathrm{Ni}, \mathrm{Pb}_{1} \mathrm{SO}_{3}, \mathrm{Sr}_{1} \mathrm{~V}_{2} \mathrm{O}_{5}, \mathrm{Zn}$, and $\mathrm{Zr}$.

Thalassionema nitzschioides in an assemblage with abundant smaller species of Coscinodiscus curvatulus, C. doljensis and bigger but mostly fragmented Thalassiosira leptopus. In the PDK dominant diatoms include Coscinodiscus oculus iridis, Thalassiosira leptopus, Coscinodiscus stellatis and Paralia sulcata.

\subsection{Mineralogy}

The mineralogical composition of the insoluble residues of the analysed rock samples is given in Table 2 . The main components of all samples are carbonate minerals (calcite and/or aragonite), clay minerals and opaline silica (biogenic opal-A with very small amounts of mineral opal-C in some samples). Opal-A (highly disordered, near amorphous) produces a single broad peak centred at approximately $4 \AA$ (Fig. 4) and opal-C (well-ordered $\alpha$-cristobalite) at 4.04-4.06 $\AA$ (JONES \& SEGNIT, 1971). All analysed samples contain quartz $\left(\mathrm{SiO}_{2}\right)$ ranging in content from $1-5 \%$. Some samples contain smaller amounts of feldspar and pyrite.

Table 4. Trace element content in Croatian diatomites ( $\mathrm{mg} / \mathrm{kg}$ ).

\begin{tabular}{|c|c|c|c|c|c|c|c|c|c|}
\hline $\begin{array}{l}\text { Sample/ } \\
\text { Element }\end{array}$ & MA-4 & PDK & D-01 & D-02B & JU-1 & OP-4 & OP-4A & MR-10 & MR-10B \\
\hline Mo & 24.4 & 17.4 & 20.2 & 14.4 & 2.2 & 2.3 & 0.8 & 98.7 & 8.0 \\
\hline $\mathrm{Cu}$ & 46.8 & 32.9 & 41.5 & 82.9 & 21.3 & 24.6 & 31.0 & 37.9 & 31.4 \\
\hline $\mathrm{Pb}$ & 19.4 & 6.9 & 14.7 & 11.8 & 5.6 & 10.8 & 4.3 & 10.4 & 12.9 \\
\hline $\mathrm{Zn}$ & 75 & 33 & 65 & 63 & 27 & 23 & 14 & 48 & 52 \\
\hline $\mathrm{Ni}$ & 113 & 81 & 89 & 81 & 31 & 35 & 13 & 133 & 62 \\
\hline Co & 21 & 6 & 16 & 8 & 5 & 5 & 2 & 11 & 5 \\
\hline As & 15 & 7 & 7 & 5 & 10 & 5 & 0.05 & 5 & 6 \\
\hline U & 8 & 10 & 6 & 4 & 5 & 8 & 6 & 36 & 8 \\
\hline Th & 8 & 3 & 6 & 4 & 2 & 2 & 1 & 3 & 3 \\
\hline $\mathrm{Sr}$ & 518 & 3405 & 427 & 549 & 3438 & 6405 & 5161 & 1745 & 1653 \\
\hline $\mathrm{Cd}$ & 0.8 & 6.2 & 0.8 & 4.5 & 0.8 & 1.1 & 0.3 & 2.9 & 1.6 \\
\hline $\mathrm{Sb}$ & 1.8 & 0.9 & 1.3 & 1.7 & 0.6 & 2.2 & 0.4 & 4.1 & 2.0 \\
\hline $\mathrm{Bi}$ & 0.5 & $<0.1$ & 0.3 & 0.2 & 0.1 & 0.2 & 0.1 & 0.2 & 0.2 \\
\hline V & 104 & 64 & 75 & 81 & 43 & 23 & 17 & 51 & 60 \\
\hline $\mathrm{La}$ & 24 & 12 & 17 & 12 & 8 & 9 & 5 & 10 & 12 \\
\hline $\mathrm{Ba}$ & 275 & 340 & 183 & 168 & 336 & 176 & 170 & 632 & 679 \\
\hline W & 1.4 & $<0.5$ & 1.2 & 0.8 & 0.5 & 0.4 & 0.2 & 0.6 & 0.6 \\
\hline $\mathrm{Ce}$ & 54 & 21 & 35 & 23 & 15 & 17 & 9 & 20 & 24 \\
\hline Sn & 2.4 & 1.0 & 1.6 & 1.3 & 0.7 & 0.8 & 0.6 & 1.1 & 1.3 \\
\hline$Y$ & 19 & 13 & 14 & 9 & 6 & 10 & 7 & 7 & 8 \\
\hline $\mathrm{Nb}$ & 6 & 4 & 5 & 4 & 2 & 2 & 1 & 3 & 4 \\
\hline $\mathrm{Ta}$ & 0.5 & 0.3 & 0.4 & 0.3 & 0.2 & 0.1 & 0.05 & 0.2 & 0.2 \\
\hline Sc & 12 & 5 & 8 & 7 & 3 & 3 & 2 & 6 & 7 \\
\hline $\mathrm{Li}$ & 40 & 23 & 35 & 29 & 14 & 12 & 9 & 25 & 30 \\
\hline Se & 5 & 3 & 4 & 3 & 2 & 3 & 3 & 11 & 4 \\
\hline $\mathrm{Zr}$ & 39 & 49 & 34 & 26 & 22 & 8 & 6 & 22 & 25 \\
\hline
\end{tabular}

Celatom ${ }^{\oplus}$ Diatomaceous Earth Functional Additive Standard Celatom ${ }^{\circledast}$ MN 51: $\mathrm{Pb}=15 \mathrm{mg} / \mathrm{kg}, \mathrm{As}=20 \mathrm{mg} / \mathrm{kg}$.

\subsection{Geochemistry}

The results of geochemical analysis for major elements are shown in Table 3. The concentration of total silica in samples varies from 3.96 to $61.91 \%$. The measured content of $\mathrm{SiO}_{2}$ in most samples is relatively small $(<47.71 \%)$ except for the samples from the borehole Podsusedsko Dolje (D-01 and D-02B; $55.58 \%$ and 61.91\% respectively). In comparison to the standard sample (Celatom ${ }^{\circledR}$ MN 51) the majority of analysed samples have a high content of $\mathrm{CaO}(>35 \%)$ and relatively high content of $\mathrm{MgO}$ (Tab. 3). The high content of carbonate component in the samples results in high values of LOI (>18.60\%).

The measured content of trace elements of nine DE samples is given in Table 4. It is significant that the concentrations of Mo and $\mathrm{Cu}$ are higher in samples from Medvednica Mt. than form elsewhere. The highest concentration of $\mathrm{Pb}(19.4 \mathrm{mg} / \mathrm{kg})$, which exceed the recommended value of $15 \mathrm{mg} / \mathrm{kg}$ for diatomaceous earth of standard sample, is determined in sample MA-4 from Medvednica Mt. (Tab. 4). The samples from Medvednica Mt. and samples MR-10 and MR-10B from Banovina region also have increased concentrations of $\mathrm{Ni}(>50 \mathrm{mg} / \mathrm{kg})$. The elevated concentrations of uranium, especially in sample MR-10 from Banovina, are connected to the higher content of organic matter.

\subsection{Insecticidal efficacy on common stored pests}

Results among our samples of inert dust efficacy against rice weevil (RW), S. oryzae are shown in Table 6., where the highest efficacy is seen in samples D-01, D-02B and MA-4.

\section{DISCUSSION}

The results of XRPD analyses showed that all analysed samples contain greater or lesser amounts of opal-A and quartz (Tab. 2). Quantitative analyses of the mineralogical composition of samples obtained by XRPD analyses (Tab. 2.) gave larger amounts of opal silica than results of calculations by Boströms' and Murdmaas' formulas (Tab. 5). These types of calculations are based on the assumption that the $\mathrm{SiO}_{2} / \mathrm{Al}_{2} \mathrm{O}_{3}$ ratio in terrigenous clay and silty clay is almost constant, and varies from 3.5 to 2.7. According to GRIZELJ et al. (2007; 2017) XRPD analyses of Miocene pelitic sediments from the Croatian part of the PBS revealed that most of the samples contain the same mineral phases, but they are present in different quantities in different samples. The ratio of $\mathrm{SiO}_{2} / \mathrm{Al}_{2} \mathrm{O}_{3}$ can vary within one clay mineral group and varies considerably within different clay mineral groups. Clay minerals have a wide range of variations in chemical composition and the variation is a composite of all the errors inherent in any 

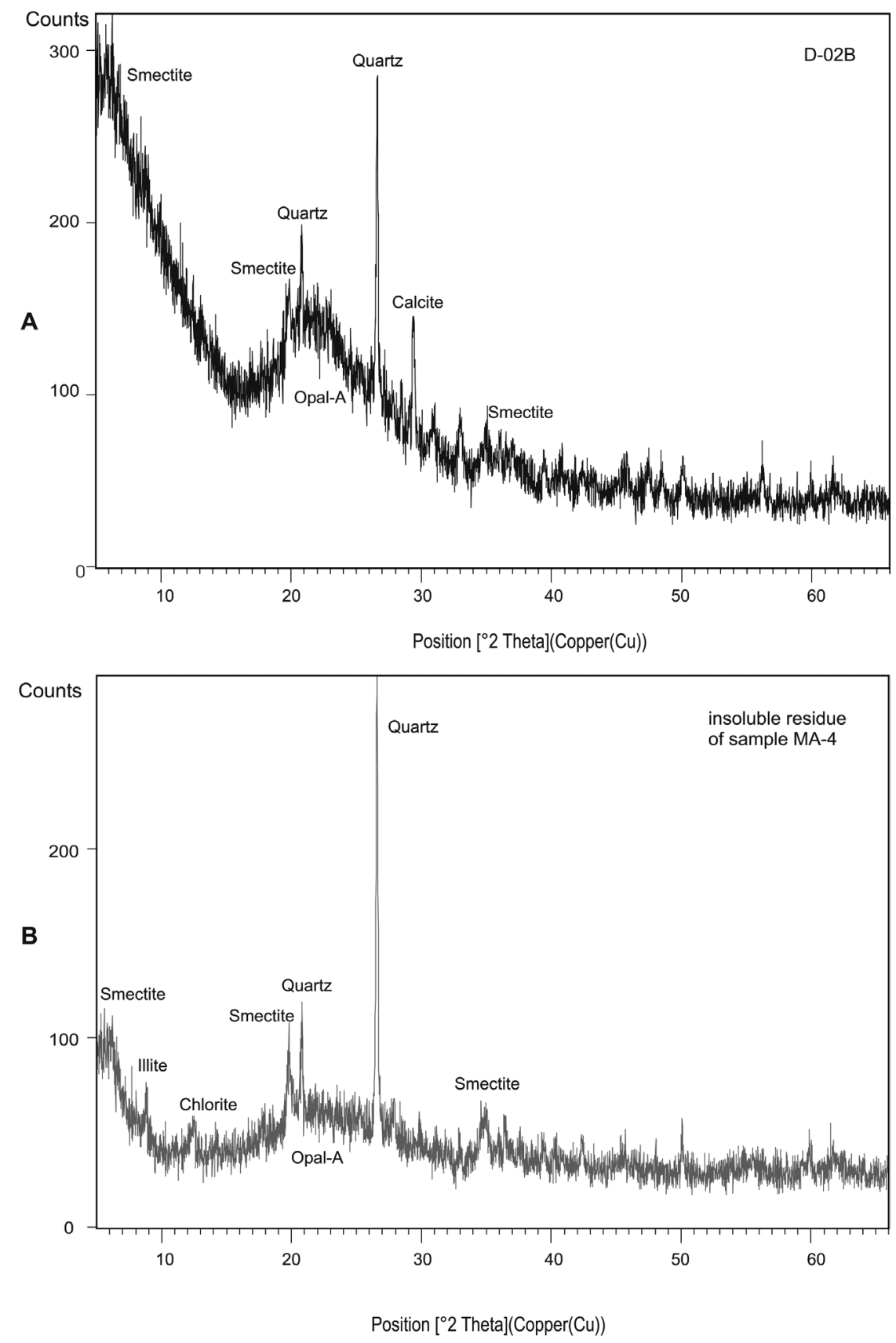

Figure 4. XRPD pattern of bulk sample D-02B, and insoluble rock residue of sample MA-4.

Table 5. Content of Opal- $\mathrm{A}^{\mathrm{B}}$ according to standard Boströms'formula (BOSTRÖM at al., 1972) and Opal- $\mathrm{A}^{\mathrm{M}}$ according to modified Murdmaas' formula (MURDMAA et al., 1980). $\mathrm{SiO}_{2}$ and $\mathrm{Al}_{2} \mathrm{O}_{3}$ are from Table 3 .

\begin{tabular}{|c|c|c|c|c|c|c|c|c|c|}
\hline $\begin{array}{l}\text { Element/ } \\
\text { mineral }\end{array}$ & $\sum_{\Sigma}^{+1}$ & 흐 & $\overline{0}$ & 只 & $\stackrel{\ulcorner}{\supsetneq}$ & $\begin{array}{l}+ \\
\text { ò }\end{array}$ & $\begin{array}{l}\frac{\pi}{5} \\
0 \\
0\end{array}$ & $\frac{0}{\frac{1}{c}}$ & $\begin{array}{l}\infty \\
\frac{0}{\dot{1}} \\
\stackrel{1}{\Sigma}\end{array}$ \\
\hline$U_{2}$ & 7.71 & 21.26 & 5.58 & 1.91 & 18.65 & 7.37 & 3.96 & 14.21 & 16.22 \\
\hline L & & & & & & 2.32 & 1 & & 44 \\
\hline$\cdot \mathrm{Al}_{2} \mathrm{O}_{3}$ & 5.43 & נ5.5. & 20.49 & 0.90 & 9.45 & 0.90 & 3.93 & 14.19 & 16.32 \\
\hline $2.5 \cdot \mathrm{Al}_{2} \mathrm{O}_{3}$ & 23.79 & 1218 & 1814 & 14.34 & 8.50 & 6.26 & 3.54 & 12.77 & 14.69 \\
\hline Opal-A ${ }^{B}$ & 1.28 & 7.73 & 35.09 & 45.98 & 9.20 & 0.41 & 0.03 & 0.02 & -0.10 \\
\hline & 3.92 & 9.08 & 37.14 & 47.57 & 10.15 & 1.11 & 0.42 & 1.44 & 1.53 \\
\hline
\end{tabular}

analytic analysis: operator, instrument, method, beneficiation, sampling (WEAWER and POLLARD, 1973; WEAVER 1989).

According to the geochemical analyses there is a significant variation in the total silica $\left(\mathrm{SiO}_{2}\right)$ content in samples collected on
Table 6. Efficacy of 9 Croatian inert dusts and standard Celatom ${ }^{\circledR}$ MN 51 against Sitophilus oryzae (L.) adults after 7 days of exposure with $\mathrm{LD}_{50} / \mathrm{LD}_{90}$ values $(95 \%$ fiducial limits).

\begin{tabular}{|c|c|c|}
\hline \multirow{2}{*}{ Inert dust } & \multicolumn{2}{|c|}{ Lethal doses (ppm) } \\
\hline & LD50* & LD90* \\
\hline D-01 & $156.5(62.28-213.26)$ & $447.2(419.88-481.90)$ \\
\hline $\mathrm{D}-02 \mathrm{~B}$ & $300 \mathrm{ppm}=82.5 \%$ & $359.6(240.55-422.56)$ \\
\hline JU-1 & $351.4(324.22-373.43)$ & $606.7(575.90-648.08)$ \\
\hline MA-4 & $232.2(183.05-266.05)$ & $458.7(436.21-487.52)$ \\
\hline MR-10 & $693.3(646.91-778.77)$ & 954.1 (847.50-1159.46) \\
\hline MR-10B & $588.6(564.40-623.79)$ & 831.1 (763.36-944.09) \\
\hline $\mathrm{OP}-4$ & $421.5(346.71-460.50)$ & $867.7(750.76-1161.96)$ \\
\hline OP-4A & $534.8(517.44-555.17)$ & 744.4 (699.14-813.40) \\
\hline PD-1 & $362.8(314.80-391.50)$ & 726.4 (668.39-816.94) \\
\hline Celatom ${ }^{\oplus}$ MN 51 & $300 \mathrm{ppm}=83.5 \%$ & $334.1(86.64-399.00)$ \\
\hline
\end{tabular}

*LC50 and LD90 expressed as parts per million (ppm), Confidence limits (CL) are given in parentheses 
the Medvednica Mt. (21.26\% sample PDK, $47.71 \%$, sample MA4, and $61.91 \%$ sample D-02B) (Tab. 3). This is particularly evident at the Podsusedsko Dolje site, where the distance from the sample PDK and samples D-01 and D-02B is about a kilometre. Laterally marked variations in the silica content of the investigated diatomites are most likely the result of the marine geomorphology of the depth and its variations during the mid-Sarmatian, as well as diagenesis and tectonic processes that modify the composition and crystalline phase of the mineral. This also applies to the $\mathrm{SiO}_{2}$ content at the Jurjevčani (18.65\%) site (Žumberak Mt.), which is significantly lower than the average silica content on the Medvednica Mt. (mean $=48.63 \%$ ) considering that the direct distance from the Podsusedsko Dolje site is only $25 \mathrm{~km}$ today. This is in accordance with their origin where the most prospective diatomites from Medvednica Mt. belong to the upwelling zone of the temperate climate with seasonal blooming (GALOVIĆ \& BAJRAKTAREVIĆ, 2006), while that from Žumberak belongs to a more coastal marine area. The concentration of silica in the samples from Banovina is lower than that registered in the samples from the Medvednica and Žumberak Mts. (Tab. 3). The lowest content of $\mathrm{SiO}_{2}$ is found in the samples from Slavonia (OP: $\leq 7.37 \%$ ) which is why we advocate tripolite instead of ordinary diatomite. The $\mathrm{SiO}_{2}$ concentrations in those analysed samples are more depleted in comparison with the standard Celatom ${ }^{\circledR} \mathrm{MN}-51$. The concentration ranges from 3.96 to $7.37 \% \mathrm{SiO}_{2}$ (Tab. 3). The insecticidal effect of DEs against stored product insects greatly depends on the amount of biogenic silica (KORUNIĆ, 2013). Samples with the lowest concentration of silica have the highest carbonate component $(\mathrm{CaO})$ content. According to FIELDS \& MUIR (1995) calcium oxide also has some insecticidal activity. Samples JU, OP and MR have enhanced $\mathrm{CaO}$ (36.90-49.26\%) which could be a reason why they showed promising insecticidal efficacy in preliminary testing. All samples except D- 01 and D- $02 B$ have much higher contents of $\mathrm{CaO}$ compared to the standard sample (Tab. 3). The $\mathrm{MgO}$ content is in most samples associated with the $\mathrm{CaO}$ content which is probably due to the originally precipitated dolomite in association with calcite. The content of alumina depends on the amount of the clay component in the sample. In relation to Celatom ${ }^{\circledR}$ MN 51 only one analysed sample, MA-4, has a slightly elevated content of alumina $\left(\mathrm{Al}_{2} \mathrm{O}_{3}\right)$, which is probably a part of the detected smectite which increased its adsorption (Tab. 2 and 3). The $\mathrm{Fe}_{2} \mathrm{O}_{3}$ content is slightly higher in all analysed samples from the Medvednica Mt. as well as in the sample MR-10B (Banovina) in comparison to Celatom ${ }^{\circledR}$ MN 51. The highest concentration of iron registered in the sample MA-4 from the Medvednica Mt. probably originates from the chlorite mineral group occurring in the sample (Tab. 2). The sum of all other oxides $\left(\mathrm{Na}_{2} \mathrm{O}, \mathrm{K}_{2} \mathrm{O}+\right.$ $\mathrm{MnO}+\mathrm{TiO}_{2}+\mathrm{P}_{2} \mathrm{O}_{5}$ ) in the analysed samples of Croatian diatomites is smaller than their content in Celatom ${ }^{\circledR} \mathrm{MN} 51$ (Tab. 3). Among the trace elements, only the concentrations of potentially toxic lead $(\mathrm{Pb})$ and arsenic are of importance. The amount of $\mathrm{Pb}$ exceeds the limit values relative to Celatom ${ }^{\circledR}$ MN $51(15 \mathrm{mg} / \mathrm{kg})$ solely in the sample MA-4 (19.4 mg/kg) from Medvednica Mt., while the concentrations in all other samples are lower. Enhanced lead might be a result of the weathering of the galenite ore deposit there. The content of arsenic (As) is significantly lower in all analysed samples compared to sample Celatom ${ }^{\circledR} \mathrm{MN} 51$ (Tab. 4).

The highest efficacy against the rice weevil, $S$. oryzae showed in samples D-01, D-02B and MA-4 (Tab. 6). Samples D-01 and D-02B have smaller amounts of quartz (4-5\%) and maximum amount of opal-A (41-50\%), as BSi, which make them suitable for commercial usage as DE (Tab. 2, Fig. 4). As a BSi component,
Thalassionema nitzschioides dominate in the assemblage with abundant, bigger species $(65-80 \mu \mathrm{m})$ of Coscinodiscus group (Coscinodiscus oculus iridis, Thalassiosira leptopus, Coscinodiscus radiatus, Coscinodiscus stellatis, Coscinodiscus rothii, Coscinodiscus perforatus var. cellulosus), which are mostly fragmented, and with their smaller representatives (Coscinodiscus curvatulus, Coscinodiscus doljensis, Coscinodiscus sarmaticus, Coscinodiscus rugulosus, Paralia sulcata), giving better efficacy of D-02B sample as DE like MA-4 (Fig. 3, Tab. 1). The relatively high content of opal-A (28\%) and a small amount of quartz (5\%) characterised sample MA-4 (Tab. 2). In previous testing (LIŠKA et al., 2015) of samples (MA-4, MR-10, MR-10B, OP-4, OP-4A and PD1) against Tribolium castaneum (HERBST) and Rhyzopertha dominica (FAB.) the highest efficacy ( 7 and 14 days after treatment) showed in sample MA-4, reaching T. castaneum mortality of $61.50 \%$ and $99 \%$, respectively, and $25.50 \%$ and $34.0 \%$, respectively against $R$. dominica. In addition, there were no statistical differences of mortality rates with the standard Celatom ${ }^{\circledR}$ MN 51. According to ZIAEE et al. (2013) each diatom species could have its own mode of physical action, regarding the number and size of pores and distribution of striae and if it is greater, the insecticidal activity would be higher. The results on diatom morphometrics show that the most efficient diatomites consist of midsized $(<45 \mu \mathrm{m}$ ) Coscinodiscus (Coscinodiscus curvatulus and $C$. doljensis) with numerous smaller pores, and lancet shaped Thalassionema nitzschioides that make up its active surface (Fig. 3). This is in agreement with KORUNIĆ (1998), that diatoms with discoid, flattened and linear body shapes better cover insect cuticle in relation to diatoms of cylindrical and round shapes. DE particles can effectively adsorb epicuticular lipids from the cuticle surface of the treated insects (ROHITHA PRASANTHA et al., 2015) and these phenomena may have a great effect on efficacy against insects. According to KORUNIĆ et al. (2016), larger available active surfaces have a higher sorption capacity for the lipids and are more effective desiccants. In the experiment conducted by KORUNIĆ \& FIELDS (2016) silica aerogel Sipernat $50 \mathrm{~S}$ with oil sorption of $3.23 \mathrm{ml} / \mathrm{g}$ dust was significantly more effective against Sitophilus granarius (LINNAEUS) in comparison with other DEs with oil sorption from 1.13 to $1.96 \mathrm{ml} / \mathrm{g}$ dust. Inert dusts MA-4 with oil sorption of 1.23 and Celatom ${ }^{\circledR}$ MN 51 with oil sorption of 1.20 were equally effective against rice weevil Sitophilus oryzae (LINNAEUS) and granary weevil, S. granarius. Nevertheless, noticeable sponge spicules and silicoflagellates in samples may damage the digestive tract or breathing organ, also resulting in internal desiccation or suffocation of the insects (JACKSON \& WEBLEY, 1994), which increases efficacy as well. Sample MR-10 from Banovina region has only $3 \%$ opal-A, while sample MR-10B did not contain opaline silica. Two samples from Slavonia OP-4 and OP-4A contain small amounts of opal-C (Tab. 2). Based on the palaeontological results, the sporadic appearance of sponge spicules was noticed only in samples of OP, which is in agreement with the results of Murdmaas' modified formula (Tab. 5). These samples are partially modified by calcinations in the process of diagenesis. It is well known that during diagenesis siliceous deposits undergo mineralogical changes from non-crystalline biogenic silica (opal-A) to microcrystalline opal (opal-CT/C) to microcrystalline quartz $\left(\mathrm{SiO}_{2}\right)$ (WILLIAMS et al., 1985; FLÖRKE et al., 1991; CADY et al., 1996; LYNNE \& CAMPBELL, 2004). Micro-crystalline silica minerals (quartz) have been shown to be carcinogenic if inhaled. In addition, crystalline silica is classified as carcinogenic to humans by the International Agency for Research on Cancer (IARC, 1997). DEs registered as 
insecticides generally have less than $6 \%$ of quartz, like all analysed samples. However, the use of a proper dust mask, or the use of low crystalline silica DE can protect against this health risk (DESMARCHELIER \& ALLEN, 2000). Very small amounts of opal-C were contained in samples PDK and D-01 (Tab. 2). Sample PDK appeared to have the best outcome on RW with inert dust less than 25 microns even after 5 days. This is in agreement with the fact that smaller particles of DEs $(<45 \mu \mathrm{m})$ are significantly more effective than larger particles (KORUNIĆ et al., 2011). Species are mostly smaller than usual, but this is in accordance with their origin in the upwelling zone and with seasonal changes during the Sarmatian, that limited their rapid growth in the blooming period (JURILJ, 1957; GALOVIĆ \& BAJRAKTAREVIĆ, 2006). Nevertheless, sample PDK has a high content of carbonate minerals (calcite and aragonite), and a smaller amount of opal-A (12\%), and quartz (3\%) (Tab. 2). Despite lower concentrations of amorphous silica in samples MA-4 $(28 \%)$ and PDK $(12 \%)$, in combination with the increased content of smectite gives even better absorption properties. Clays are also known as inert dusts like DEs (MACELJSKI \& KORUNIĆ, 1972; BANKS \& FIELDS, 1995; SUBRAMANYAM \& ROESLI, 2000) that also influenced better adsorptions here. In almost all the analysed samples smectite is the dominant clay mineral. It has a 2:1 layer structure, with a large base exchange capacity (60-130 meq/100 g) and will readily adsorb cations such as $\mathrm{H}^{+}, \mathrm{Na}^{+}, \mathrm{Ca}^{2+}$, and $\mathrm{Mg}^{2+}$ (WEAVER, 1989). Interlayer cations are hydrated and replaceable, resulting in the swelling and dehydration characteristic for smectite (SLOVENEC \& BERMANEC, 2003).

\section{CONCLUSION}

Sarmatian diatomites from Medvednica Mt. have different physical properties and efficacy on grain store insects. The analysed Croatian diatomites have significantly lower concentration of silica in relation to the standard DE. Diatomites from borehole (D-01, D-02) have the highest amorphous silica component (41$50 \%$ ) and small amounts of quartz (4-5\%) which makes them suitable for commercial usage as DE. They exhibit the highest efficacy on stored products insect rice weevil S. oryzae, together with inert dust from Medvednica Mt. (MA-4, PDK). Based on palaeontological results the most efficient analysed DE consists of mid-sized planktonic Coscinodiscus group of species where Thalassionema nitzschioides dominate which is positively correlated with their absorption. Even much smaller concentrations of amorphous silica in sample MA-4 (28\%) and PDK (12\%), but in combination with increased content of smectite gives even slightly better absorption property. The higher level of $\mathrm{CaO}(9.63-36.29)$ in correlation with Celatom ${ }^{\circledR}$ MN 51 (5.60) probably also increased efficacy. Ca (II) ions are known to improve flow rate in modified diatomite (BAZHAL et al., 1975). Probably because of all mentioned parameters diatomite MA-4 and smaller fraction of PDK show no differences of mortality rates with the standard Celatom ${ }^{\circledR} \mathrm{MN} 51$. Unfortunately, the small thicknesses of all prospective Croatian diatomites (approx. $48 \mathrm{~cm}$ ) and the slightly higher content of lead in MA-4 sample make them inadequate for classical usage as DE (MA-4= 19.4. mg/kg, Tab. 4). According to the standard sample Celatom ${ }^{\circledR}$ MN 51 the allowed maximum $\mathrm{Pb}$ concentration is $15 \mathrm{mg} / \mathrm{kg}$. The content of quartz in the samples is $1-5 \%$. Concerning insecticidal effects against the rice weevil, $S$. oryzae and taking into account the small thicknesses of all prospective Croatian diatomite layers, there is the possibility of mixing these inert dusts with some other natural compounds, with other insecticide effects, to provide the same (or even higher) insecticidal level.

\section{ACKNOWLEDGEMENT}

Financial support for this research was provided by the Croatian Science Foundation (HRZZ) through the scientific research project IP-11-2013-5570 (project leader Prof. dr. sc. Vlatka Rozman): Development of new natural insecticide formulations based on inert dusts and botanicals to replace synthetic, conventional insecticides, http://www.diacromixpest.eu/. The authors are grateful to Snježana MIHALIĆ ARBANAS, Faculty of Mining, Geology and Petroleum Engineering, for allowing using Dolje borehole samples for the purpose of this research. We would like to thank to Stjepan ĆORIĆ (GBA, Austria) and anonymous reviewer as well for their valuable comments and suggestions that improve the manuscript.

\section{REFERENCES}

BAJRAKTAREVIĆ, Z. (1984): The application of microforaminiferal association and nannofossils for biostratigraphic classification of the Middle Miocene of $\mathrm{N}$. Croatia.- Acta Geologica, 49/1, 1-34.

BAJRAKTAREVIĆ, Z., BLAŠKOVIĆ, I. \& POLŠAK, A. (1986): Tripoli-stratigrafski položaj, paleontološka i sedimentološka obilježja u području Tethysa [TripoliStratigraphic position, paleontologic and sedimentologic characteristics in Tethys area - in Croatian].- 11. Kongr. Geol. Jugosl., 1986/2, 319-331, Tara.

BANKS, J. \& FIELDS, P. (1995): Physical methods for insect control in stored grain ecosystems.- In: JAYAS, D.S., WHITE, N.D.G. \& MUIR, W.E. (eds.): Stored Grain Ecosystems, Marcel Dekker, New York, 353-409.

BAZHAL, I.G., VORONA, L.G., LESHCHENKO, A.V. \& PEREVERZEVA, I.N. (1975): Effect of chemical modification of diatomite powder on their filtration qualities.- Sov. Prog. Chem., 41, 91-92.

BOSTRÖM, K., JOENSUU VALDES, S. \& RIERA, M. (1972): Geochemical history of South Atlantic Ocean sediments since late cretaceous.- Mar. Geol. 12, 85-121. doi: 10.1016/0025-3227(72)90023-0

CADY, S.L., WENK, H.R. \& DOWNING, K.H. (1996): HRTEM of microcrystalline opal in chert and porcelanite from the Monterey Formation, California.-American Mineralogist, 81, 1380-1395. doi: 10.2138/am-1996-11-1211

DESMARCHELIER, K.M. \& ALLEN, S.E. (2000): Diatomaceous earth: health, safety, environment, residues and regulatory issues.- In: JIN, Z., LIANG, Q., LIANG, Y., TAN, X. \& GUAN, L. (eds.): Proceedings 7th International Working Conference on Stored-product Protection, Beijing, PR China, 309-312. ISBN 7536440987

DOLLEY, T.P. \& MOYLE, P.R. (2003): History and overview of the U.S. diatomite mining industry, with emphasis on the Western United States.- US Geological Survey Bulletin, 2209, E1-E8.

EBELING, W. (1971): Sorptive Dusts for Pest Control.-Annual Review of Entomology, 16(1), 123-158. doi: 10.1146/annurev.en.16.010171.001011

EBERL, D.D. (2003): User's guide to RockJock-A program for determination quantitative mineralogy from powder X-ray diffraction data.- U.S. Geological Survey, Bulder, Colorado, $46 \mathrm{p}$.

FIELDS, P. (2003): Laboratory selection for resistance to diatomaceous earth.- In: CREDLAND, P.F. ARMITAGE, D.M., BELL, C.H. COGAN, P.M. \& HIGHLEY, E. (eds.): Proceedings of the 8th international working conference on stored product protection, York, UK, 776-778.

FIELDS, P.G. \& MUIR, W.E. (1995): Physical control.- In: SUBRAMANYAM, B. \& HAGSTRUM, D.W (eds.): Integrated pest management of insects in stored products, Marcel-Dekker Inc., 195-222, New York.

FLÖRKE, O.W., GRAETSCH, H., MARTIN, B., RÖLLER, K. \& WIRTH, R. (1991): Nomenclature of microcrystalline and non-crystalline silica minerals, based on structure and microstructure.- Neues Jahrbuch für Mineralogie-Abhandlungen, $163 / 1,19-42$.

GALOVIĆ, I. (2001): Silicous phytoplankton assemblage from Sarmatian beds in the Markuševec area (Mt. Medvednica, Croatia).-Acta Botanica Croatica, 60, 1-10.

GALOVIĆ, I. \& BAJRAKTAREVIĆ, Z. (2006): Sarmatian biostratigraphy of the Mt. Medvednica at Zagreb based on siliceous microfossils (North Croatia, Central Paratethys).- Geologica Carpathica, 57, 199-210.

GALOVIĆ, I., HALAMIĆ, J., ROZMAN, V., KORUNIĆ, Z., LIŠKA, A., BALIČEVIĆ, R. \& LUCIĆ, P. (2015): Dijatomiti u Hrvatskoj: njihov potencijal kao prirodni insekticid. [Diatomite in Croatia: their potential as natural insecticide - in Croatian].- In: HORVAT, M. \& WACHA, L. (eds.): 5th Croatian Geological Congress, Abstract book, 83-84.

GORJANOVIĆ-KRAMBERGER, D. (1908): Geologijska pregledna karta Kraljevine Hrvatske - Slavonije. Tumač geologijskoj karti Zagreb [Geologic schematic map of the Kingdom of Croatia-Slavonia. Explanatory notes for the geologic map Zagreb - in Croatian].-Nakl. Kralj. zemalj. vlade, Odjel za unutar. poslove, Zagreb, $75 \mathrm{p}$. 
GRIZELJ, A., PEH, Z., TIBLJAŠ, D., KOVAČIĆ, M. \& KUREČIĆ, T. (2017): Mineralogical and geochemical characteristics of Miocene pelitic sedimentary rocks from the south-western part of the Pannonian Basin System (Croatia): Implications for provenance studies.- Geoscience Frontiers, 8/1, 65-80. doi: org/10.1016/j. gsf.2015.11.009

GRIZELJ, A., TIBLJAŠ, D. \& KOVAČIĆ, M. (2007): Mineralogy and geochemistry of Upper Miocene pelitic sediments of Zagorje Basin (Croatia): Implication for evolution of Pannonian Basin.- Geologica Carpathica, 58/3, 263-276.

HAJÓS, M. (1986): Stratigraphy of Hungary's Miocene diatomaceous Earth deposits.Geologica Hungarica, Series Paleontologica, fasc., 49, $339 \mathrm{p}$.

HARWOOD, D.M. (2010): Diatomite.- In: SMOL, J.P. \& STOERMER, E.F. (eds.): The Diatoms: Applications for the Environmental and Earth Sciences, Cambridge University Press, 570-574. doi: 10.1017/CBO9780511763175.034

HORVAT, A. (2004): Middle Miocene siliceous algae of Slovenia: paleontology, stratigraphy, paleoecology, paleobiogeography.-Založba ZRC, Ljubljana, ZRC SAZU, $255 \mathrm{p}$.

HORVÁTH, F. \& ROYDEN, L.H. (1981): Mechanism for the formation of the IntraCarpathian Basins: A Rewiev.- Earth Evolution Sciences, 1, 307-316.

IARC - International Agency for Research on Cancer (1997): IARC monographs on the evaluation of carcinogenic risks to humans, Silica, some silicates, coal dust and para-Aramid Fibrils, 68 IARC Lyon.

IBM Corp. Released (2013): IBM SPSS Statistics for Windows, Version 22.0. Armonk, NY: IBM Corp.

JACKSON, M.L. (1956): Soil chemical analysis.--Advanced course. Medison. Wisconsin, published by the author, $894 \mathrm{p}$.

JACKSON, K. \& WEBLEY, D. (1994): Effects of Dryacide on the physical properties of grains, pulses and oilseeds.- In: HIGHLEY, E., WRIGHT, E.J., BANKS, H.J. \& CHAMP, B.R. (eds.): Stored Product Protection, Proceedings of the 6th International Working Conference on Stored-Product Protection, 635-637.

JONES, J.B. \& SEGNIT, E.R. (1971): The nature of opal 1. Nomenclature and constituent phases.- Journal of the Geological Society of Australia, 18, 1, 57-68. doi: $10.1080 / 00167617108728743$

JURILJ, A. (1957): Dijatomeje Sarmatskog mora okoline Zagreba [Diatoms from Sarmatian sea in the surroundings of Zagreb - in Croatian].- In: UGRENOVIĆ, A. (ed.): Acta Biologica I, JAZU, 28, 5-134.

KLJAJIĆ, P. \& PERIĆ, I. (2005). Resistance of stored-product insects to insecticides.Pesticidi i fitomedicina, 20, 9-28.

KOCHANSKY-DEVIDÉ, V. \& BAJRAKTAREVIĆ, Z. (1981): Miocen (baden i sarmat) najzapadnijeg ruba Medvednice [Miocene (Badenian and Sarmatian) of the westernmost margin of Medvednica Mt. - in Croatian].- Geološki vjesnik, 33, 43-48. Zagreb.

KORUNIĆ, Z. (1998): Review Diatomaceous earths, a group of natural insecticides.Journal of Stored Products Research, 34/(2-3), 87-97.

KORUNIĆ, Z. (2013): Diatomaceous Earths-Natural Insecticides.- Pestic. Phytomed., 28/2, 77-95. doi: 10.2298/PIF1302077K

KORUNIĆ, Z. \& FIELDS, P.G. (2016): Relationship between insecticidal efficacy and oil absorption capacity of inert dusts against Sitophilus spp.- Indian Journal of Entomology, 78, 108-113. doi: 10.5958/0974-8172.2016.00031.6

KORUNIĆ Z., ROZMAN, V., HALAMIĆ, J., KALINOVIĆ, I. \& HAMEL, D. (2011): Insecticide potential of diatomaceous earth from Croatia.- Integrated Protection of Stored Products IOBC/WPRS Bulletin, 69, 389-397.

KORUNIĆ, Z., ROZMAN, V., LIŠKA, A. \& LUCIĆ, P. (2016): A review of natural insecticides based on diatomaceous earths.- Agriculture, 22, 10-18.

KOVÁČ, M., NAGYMAROSY, A., HOLCOVA, K., HUDÁČKOVÁ, N. \& ZLINSKA, A. (2001): Paleogeography, paleoecology and eustacy: Miocene 3rd order cycles of relative sea-level changes in the Western Carpathian-North Pannonian basins.Acta Geologica Hungarica, 44, 1-45.

LIŠKA, A., ROZMAN, V., KORUNIĆ, Z., HALAMIĆ, J., GALOVIĆ, I., LUCIĆ, P. \& BALIČEVIĆ, R. (2015): The potential of Croatian diatomaceous earths as grain protectant against three stored-product insects.- Integrated Protection of Stored Products IOBC-WPRS Bulletin, 111, 107-113.

LYNNE, B.Y. \& CAMPBELL, K.A. (2004): Morphologic and mineralogic transitions from opal-A to opal-CT in low-temperature siliceous sinter diagenesis, Taupo volcanic zone, New Zealand.- Journal of Sedimentary Research, 74/4, 561-579. doi: $10.1306 / 011704740561$

MACELJSKI, M. \& KORUNIĆ, Z. (1972): Contribution to the knowledge of the mechanism of acting of inert dusts against insects.- Plant Protection, 22, 117-118, 377-387.

MURDMAA, I., GOEDEEV, V., KUZMINA, T., TURANSKAYA, N. \& MIKHAILOV, M. (1980): Geochemistry of the Japan trench sediments recovered on deep sea drilling project Legs 56 and 57.- In: LEE, M. \& STOUT, L.N. (eds.): Initial Reports of the Deep Sea Drilling Project, 56-57, 1213-1232. doi:10.2973/dsdp. proc.5657.154.1980

PANTOCSEK, J. (1903): Beiträge zur Kenntniss der Fossilen Bacillarien Ungarns.Nagytapolcsany-Pozsony, Teil 1, 76 p., Berlin.
PAVELIĆ, D. (2001): Tectonostratigraphic model for the North Croatian and North Bosnian sector of the Miocene Pannonian Basin System.- Basin Research, 13, 359-376. doi: 10.1046/j.0950-091x.2001.00155.x

PAVELIĆ, D., AVANIĆ, R., KOVAČIĆ, M., VRSALJKO D. \& MIKNIĆ, M. (2003): An outline of the evolution of the Croatian part of the Pannonian Basin system.In: VLAHOVIĆ, I. \& TIŠLJAR, J. (eds.): Evolution of Depositional Environments from the Paleozoic to the Quaternary in the Karst Dinarides and the Pannonian Basin.- IAS Field Trip Guidebook, 155-161, Zagreb.

ŘEHÁKOVÁ, Z. (1977): Marine planktonic diatom zones of the Central Paratethys Miocene and their correlation.- Bull. Geol. Survey Prague, 52, 147-157.

RIGAUX, M., HAUBRUGE, E. \& FIELDS, P.G. (2001): Mechanisms for tolerance to diatomaceous earth between strains of Tribolium castaneum (Coleoptera: Tenebrionidae).- Entomol. Exp. Appl., 101, 33-39.

ROETZEL, R., ĆORIĆ, S., GALOVIĆ, I \& RÖGL, F. (2006): Early Miocene (Ottnanghian) coastal upwelling conditions along the southeastern scarp of the Bohemian Massif (Parisdorf, Lower Austria, Central Paratethys).- Beitr. Paläont., 30, $387-413$.

RÖGL, F. (1996): Stratigraphic correlation of the Paratethys Oligocene and Miocene.Mitteilungen der Gesellschaft der Geologie und Bergbaustudenten Österreich, 41, $65-73$.

RÖGL, F. (1998): Paleogeographic Considerations for Mediterranean and Paratethys Seaways (Oligocene to Miocene).- Ann. Naturhist. Mus. Wien, 99, ser. A, 279-310.

ROHITHA PRASANTHA, B.D., REICHMUTH, CH., ADLER C. \& FELGENTREU, D. (2015): Lipid adsorption of diatomaceous earths and increased water permeability in the epicuticle layer of the cowpea weevil Callosobruchus maculatus (F.) and the bean weevil Acanthoscelides obtectus (Say) (Chrysomelidae).- Journal of Stored Products Research, 64, 36-41. doi: 10.1016/j.jspr.2015.08.003

ROJHT, H., HORVAT, A., ATHANASSIOU, C.G., VAYIAS, B.J., TOMANOVIĆ, Ž. \& TRDAN, S. (2010): Impact of geochemical composition of diatomaceous earth on its insecticidal activity against adults of Sitophilus oryzae (L.) (Coleoptera: Curculionidae).- J. Pest Sci., 83, 429-436. doi: 10.1007/s10340-010-0313-6

ROZMAN, V., KORUNIĆ, Z., HALAMIĆ, J., LIŠKA, A., BALIČEVIĆ, R., GALOVIĆ, I. \& LUCIĆ, P. (2015): Development of new natural insecticide formulations based on inert dusts and botanicals to replace synthetic, conventional insecticides-presentation of the research project of Croatian science foundation.- In: KORUNIĆ, J. (ed.): 27. Seminar DDD i ZUPP, Proccedings, 197-201, Zagreb.

ROZMAN, V., KORUNIĆ, Z., HALAMIĆ, J., LIŠKA, A., BALIČEVIĆ, R., GALOVIĆ, I. \& LUCIĆ, P. (2016): Second year of research project of Croatian Science Foundation on development of new formulations of natural insecticides - DIACROMIXPEST.- In: KORUNIĆ, J. (ed.): 28. Seminar DDD i ZUPP, Proccedings, 265-269, Zagreb

SCHRADER H.J. \& GERSONDE, R. (1978): Diatoms and silicoflagellates.- In: ZACHARIASSE, W.J., RIEDEL, W.R., SANFILIPPO, A., SCHMIDT, R.R., BROLSMA, M.J., SCHRADER, H.J., GERSONDE, R., DROOGER, M.M. \& BROEKMAN, J.A. (eds.): Micropaleontological counting methods and tehniques-an expercise on an eight metres section of the Lower Pliocene of Capo Rossello, Sicily, Utrecht Micropaleontological Bulletins, Schotanus \& Jens, 17, 129-176.

SCHÜTZ, K., HARZHAUSER, M., RÖGL, F., ĆORIĆ S. \& GALOVIĆ, I. (2007): Foraminiferen und Phytoplankton aus dem unteren Sarmatium des südlichen Wiener Beckens (Petronell, Niederösterreich).- Jahrbuch der Geologischen Bundesanstalt Wien, 147, 449-488.

SLOVENEC, D. \& BERMANEC, V. (2003): Sistematska minerlogija - mineralogija silikata [Systematic mineralogy - Mineralogy of silicates - in Croatian].Udžbenici Sveučilišta u Zagrebu, 359 p., Zagreb.

STARKEY, H.C., BLACKMON, P.D. \& HAUFF, P.L. (1984): The Routine Mineralogical Analysis of Clay-Bearing Samples.- U.S. Geological Survey Bulletin, 1563, 31 p., Washington.

STEININGER, F.F., MÜLLER, C. \& RÖGL, F. (1988): Correlation of Central Paratethys, Eastern Paratethys, and Mediterranean Neogene Stages.- In: ROYDEN, L.H. \& HORVATH, I.F. (eds.) The Pannonian Basins. A study in Basin Evolution. AAPG Memoir, Tulsa, 45, 79-87.

SUBRAMANYAM, B. \& HAGSTRUM, D.W. (1995). Resistance Measurement and Management.- In: SUBRAMANYAM, B. \& HAGSTRUM, D.W. (eds.): Integrated Management of Insects in Stored Products, USA: Marcel Dekker Inc., New York., 231-398.

SUBRAMANYAM, B. \& ROESLI, R. (2000): Inert dusts.- In: SUBRAMANYAM, B. \& HAGSTRUM D.W. (eds): Alternatives to Pesticides in Stored-Product IPM, Kluwer Academic Publishers, Dordrecht, 321-373. doi: 10.1007/978-1-46154353-4_12

VAYIAS, B.J., ATHANASSIOU, C.G., BUCHELOS, C.T. (2008): Evaluation of resistance development by Tribolium confusum Du Val (Coleoptera: Tenebrionidae) to diatomaceous earth under laboratory selection.- J. Stored Prod Res., 44, 162-168. doi: $10.1016 /$ j.jspr.2007.09.001

VILIČIĆ, D. (2003): Fitoplankton u ekološkom sustavu mora [Phytoplankton in marine ecological system - in Croatian].- In: DRAGANOVIĆ, J.M. (ed): Školska knjiga Zagreb, 196 p., Zagreb.

VRSALJKO, D. (1997): Stratigrafija naslaga panona jugozapadnog dijela Medvednice: [Stratigraphy of Panonian sediments of SW part of Medvednica Mt. - in Croatian 
with an English summary].- Unpubl. Master Thesis, Faculty of Science, University of Zagreb, $80 \mathrm{p}$

VRSALJKO, D. (1999): The Pannonian Palaeoecology and Biostratigraphy of Mollusca from Kostanjek-Medvednica Mt., Croatia.- Geologica Croatica, 52, 9-27. doi: 10.4154/GC.1999.02

VRSALJKO, D. (2003): Biostratigrafija miocenskih naslaga Žumberačkog i Samoborskog gorja na temelju mekušaca [Biostratigraphy of Miocene deposits from Žumberak and Samobor Mts. on the basis of molluscs - in Croatian, with an English summary].- Unpubl. PhD Thesis, Faculty of Science, University of Zagreb, $147 \mathrm{p}$.

VRSALJKO, D., PAVELIĆ, D. \& BAJRAKTAREVIĆ, Z. (2005): Stratigraphy and Palaeogeography of Miocene Deposits from the Marginal area of Žumberak Mt. and
Samoborsko gorje Mt. (Northwestern Croatia).- Geologica Croatica, 58, 133-150. doi: 10.4154/GC.2005.07

WEAVER, C.E. (1989): Clays, Muds, and Shales.- Elsevier Amsterdam, 721 p.

WEAVER, C.E. \& POLLARD, L.D. (1973): The chemistry of clay minerals. Developments in Sedimentology 15. Elsevier, Amsterdam, 213 p.

WEISS, N.L. (1985): Mineral Processing Handbook 3.- SME, New York, 9-29 p.

WILLIAMS, L.A., PARKS, G.A. \& CRERAR, D.A. (1985): Silica diagenesis, I. Solubility controls.- Journal of Sedimentary Petrology, 55/3, 301-311.

ZIAEE, M., MOHARRAMIPOUR, S. \& DADKHAHIPOUR, K. (2013): Effect of particle size of two Iranian diatomaceous earth deposits and a commercial product on Sitophilus granaries (Col.: Dryophthoridae).- Journal of Entomological Society of Iran, 33/2, 9-12. 